Ceci est un texte bilingue. La version française suit l'anglais.

\title{
CAML Renewal Task Force \\ Executive Summary
}

\section{Our Journey}

The CAML Renewal Task Force was formed in Fall 2019 to initiate (and facilitate) the Association's strategic planning (SP) process. To date, we have completed an environmental scan (Fall 2019), conducted a survey of the membership (Winter 2020), and facilitated discussions with members at a Town Hall (April 8, 2020) and the 2020 virtual conference. This work was summarized in an Executive Summary (March 2020) and a slide deck (June 2020). In 2021 we facilitated two Town Hall discussions of a draft Strategic Directions document (March 16 \& 17, 2021). For the June 2021 CAML virtual conference, we now present this updated Executive Summary and Strategic Directions. In the "Further Reading" section below we have highlighted important resources that guided our work.

\section{Pandemic}

Surprisingly, the COVID-19 global pandemic has helped focus CAML members' thoughts on what they need from, and value about, CAML. Members working from home in isolation have re-discovered the importance of the CAML community, and have been forced to rethink how we interact with each other. Video conferencing tools such as Zoom have made it easier than ever to support each other across long distances. The 2020 CAML conference saw higher-than-usual registration, which shows that we can be more inclusive, accessible, and affordable in the virtual world. Over a dozen colleagues came together at the CAML coffee check-in on Zoom in January 2021.

\section{Results/Recommendations}

Our work has culminated in a set of 5 Strategic Directions. These are purposely unnumbered, as we feel they are equally important. We provide a brief description of each Direction, followed by proposed Action Items for the CAML Board to consider.

\section{Task Force Membership}

Houman Behzadi, Head Librarian, Marvin Duchow Music Library, McGill University | Scott Cowan, Information Services Librarian, University of Windsor | Rachel Gagnon, Senior Cataloguing Librarian, Library and Archives Canada | Jan Guise, Director, University of Toronto Music Library | Sean Luyk, Digital Projects Librarian, University of Alberta | Brian McMillan, Director, Western University Music Library

\section{Further Reading}

- IAML Strategic Directions

- Music Library Association Strategic Action Plans 
- Maloney, Timothy. "A Discussion Document on the Future of CAML / Document de discussion de l'avenir de l'ACBM." CAML Review 24, no. 2 (1996): 29-43.

https://caml.journals.yorku.ca/index.php/caml/article/view/3729/2928.

- $\quad$ The Partnership Journal, Vol 15 No 1 (2020) Entire Features Section on Canadian Library Associations

- Brown, Laura and Roger Schonfeld. "Scholarly Societies in the Age of COVID." Ithaka S+R. Last Modified 28 October 2020. https://doi.org/10.18665/sr.314301

\section{Groupe de travail visant le renouveau de l'ACBM Résumé}

\section{Notre démarche}

Le groupe de travail visant le renouveau de l'ACBM a été mis sur pied à l'automne 2019, pour initier (et faciliter) le processus de planification stratégique de l'Association. Jusqu'à maintenant, nous avons complété l'analyse du milieu (automne 2019), réalisé un sondage auprès des membres (hiver 2020), et tenu des discussions ouvertes avec les membres, l'une au printemps 2020 et l'autre lors de la conférence annuelle virtuelle. Ce travail est résumé dans notre Résumé de mars 2020 et dans la présentation de juin 2020.

\section{Pandémie}

De façon plutôt étonnante, la pandémie de COVID-19 a aidé les membres de l'ACBM à faire le point et à mieux cibler ce qu'ils attendent de l'ACBM et en quoi l'Association est importante pour eux. Les membres qui travaillaient de la maison, isolés chacun chez eux, ont redécouvert l'importance de la communauté de I'ACBM, et ont été forcés de repenser comment nous interagissons. Les outils de vidéo-conférence, tels que Zoom, ont facilité plus que jamais le support mutuel que l'on peut s'offrir malgré la distance. La conférence 2020 de l'ACBM a rassemblé un nombre de participants plus élevé que d'habitude, ce qui montre que nous pouvons être plus inclusifs, plus accessibles et plus abordables dans un monde virtuel. Plus d'une douzaine de collègues se sont aussi réunis pour une petite réunion sur Zoom, en janvier 2021.

\section{Résultats/Recommandations}

Notre travail a débouché sur un ensemble de 5 orientations stratégiques. Celles-ci sont, volontairement, non-numérotées, car nous pensons que ces orientations ont toutes une importance égale. Nous présentons une courte description de chaque Orientation, suivie de quelques suggestions à l'endroit du Conseil d'administration.

Membres du groupe de travail

Houman Behzadi, Bibliothécaire en chef, Marvin Duchow Music Library, McGill University | Scott Cowan, Information Services Librarian, University of Windsor | Rachel Gagnon, Bibliothécaire senior au catalogage, 
Bibliothèque et archives Canada | Jan Guise, Directrice, University of Toronto Music Library | Sean Luyk, Digital Projects Librarian, University of Alberta | Brian McMillan, Directeur, Western University Music Library

\section{Autres documents à consulter}

- IAML Strategic Directions

- Music Library Association Strategic Action Plans

- Maloney, Timothy. "A Discussion Document on the Future of CAML / Document de discussion de l'avenir de l'ACBM." Revue de l'ACBM 24, no. 2 (1996) : 29-43. https://caml.journals.yorku.ca/index.php/caml/article/view/3729/2928.

- $\quad$ The Partnership Journal, Vol 15 No 1 (2020) Comprend une section complète consacrée aux associations de bibliothécaires canadiens

- Brown, Laura et Roger Schonfeld. "Scholarly Societies in the Age of COVID." Ithaka S+R. Modifié le 28 octobre 2020. https://doi.org/10.18665/sr.314301 